\title{
DISPERSIVE SOLITONS IN MAGNETO-OPTIC WAVEGUIDES
}

\author{
Jose Vega-Guzman ${ }^{1}$, Malik Zaka Ullah ${ }^{2}$, Mir Asma ${ }^{3}$, \\ Qin Zhou ${ }^{4} \&$ Anjan Biswas ${ }^{2,5}$ \\ ${ }^{1}$ Department of Mathematics, Lamar University \\ Beaumont, TX-77710, USA \\ ${ }^{2}$ Department of Mathematics, Faculty of Science,
} King Khalid University, PO Box 9004, Abha-61413, Saudi Arabia

${ }^{3}$ Institute of Mathematical Sciences, Faculty of Science, University of Malaya, 50601 Kuala Lumpur, Malaysia

${ }^{4}$ School of Electronics and Information Engineering, Wuhan Donghu University, Wuhan, 430212, PR China

${ }^{5}$ Department of Mathematics and Statistics, Tshwane University of Technology, Pretoria-0008, South Africa

\begin{abstract}
This paper obtains bright, dark and singular dispersive optical soliton solutions with magnetooptic waveguides. The governing equation is the coupled Schrödinger-Hirota equation. The existence criteria of these solitons are also presented. Both Kerr law and power law of nonlinearity are considered.
\end{abstract}

OCIS Codes: 060.2310; 060.4510; 060.5530; 190.3270; 190.4370

Key words: solitons; magneto-optic; constraints. 


\section{INTRODUCTION}

Dispersive optical solitons occur in nature when the group velocity dispersion (GVD) is small and therefore it needs to be compensated with higher order dispersion terms [1-10]. The study of dispersive optical solitons in magneto-optic wavegiuides is being conducted in this paper for the first time. Such waveguides can compel bright solitons from a state of attraction to a state of isolation [2]. The governing equation is the coupled Schrödinger-Hirota equation (SHE). In the past, results on non-dispersive optical solitons in magneto-optic waveguides has been reported [9]. The methodology adopted in this paper is the usage of undetermined coefficients. Bright, dark and singular soliton solutions are obtained. There are two types of nonlinearity studied and they are Kerr law and its generalization to power law. The existence criteria for these solitons, also known as constraint conditions, are also given.

\section{GOVERNING MODEL}

The governing equation for the magneto-optic waveguides is [9]:

$$
\begin{gathered}
\quad i q_{t}+i \alpha_{1} q_{x}+a_{1} q_{x x}+b_{1} q_{x t}+\left\{c_{1} F\left(|q|^{2}\right)+d_{1} F\left(|r|^{2}\right)\right\} q \\
+\quad i \gamma_{1} q_{x x x}+i \lambda_{1}\left(|q|^{2} q\right)_{x}+i \xi_{1}|q|^{2} q_{x}+i \eta_{1}\left(|q|^{2}\right)_{x} q=Q_{1} r \\
\\
\quad i r_{t}+i \alpha_{2} r_{x}+a_{2} r_{x x}+b_{2} r_{x t}+\left\{c_{2} F\left(|r|^{2}\right)+d_{2} F\left(|q|^{2}\right)\right\} r \\
+\quad i \gamma_{2} r_{x x x}+i \lambda_{2}\left(|r|^{2} r\right)_{x}+i \xi_{2}|r|^{2} r_{x}+i \eta_{2}\left(|r|^{2}\right)_{x} r=Q_{2} q
\end{gathered}
$$

Here, $Q_{j}$ for $j=1,2$ are magneto-optic parameters and $a_{j}$ are the coefficients of GVD while $b_{j}$ are the spatio-temporal dispersion (STD) terms. The inclusion of STD is necessary in order to make the model well-posed as suggested during $2012[5,7]$. If GVD is small, one needs to consider third order dispersion (3OD) that is represented by the coefficient of $\gamma_{j}$. Also $\alpha_{j}$ is the coefficient of intra-modal dispersion. Then $\lambda_{j}$ gives the self-steepening term that avoids the formation of shock waves. Finally $\xi_{j}$ and $\eta_{j}$ sands for the coefficients of nonlinear dispersion. The dependent variables $q(x, t)$ and $r(x, t)$ are the two complex-valued wave profiles. In order to solve (1) and (2), the guess functions to be taken are [9]:

$$
\begin{aligned}
& q(x, t)=P_{1}(x, t) e^{i \phi(x, t)} \\
& r(x, t)=P_{2}(x, t) e^{i \phi(x, t)}
\end{aligned}
$$

where $P_{l}(x, t)(l=1,2)$ represents the amplitude component of the soliton solution while the phase component $\phi(x, t)$ is defined by

$$
\phi(x, t)=-\kappa x+\omega t+\theta .
$$

Here $\kappa$ is the frequency of the solitons while $\omega$ represents the wave number and $\theta$ is the phase constant. Substituting (4) and (5) into (??) and (??) and then decomposing into real and imaginary parts gives in a simplified form

$$
\begin{aligned}
& \left(a_{l}+3 \kappa \gamma_{l}\right) \frac{\partial^{2} P_{l}}{\partial x^{2}}+b_{l} \frac{\partial^{2} P_{l}}{\partial x \partial t}+\left[\kappa\left(\alpha_{l}+b_{l} \omega_{l}-a_{l} \kappa-\gamma_{l} \kappa^{2}\right)-\omega\right] P_{l} \\
+ & \kappa\left(\xi_{l}+\lambda_{l}\right) P_{l}^{3}+\left\{c_{l} F\left(P_{l}^{2}\right)+d_{l} F\left(P_{l}^{2}\right)\right\} P_{l}=Q_{l} P_{\bar{l}}
\end{aligned}
$$


and

$$
\begin{aligned}
& \left(1-b_{l} \kappa\right) \frac{\partial P_{l}}{\partial t}+\left(b_{l} \omega+\alpha_{l}-2 a_{l} \kappa-3 \gamma_{l} \kappa^{2}\right) \frac{\partial P_{l}}{\partial x} \\
+ & \left(2 \eta_{l}+\xi_{l}+3 \lambda_{l}\right) P_{l}^{2} \frac{\partial P_{l}}{\partial x}+\gamma_{l} \frac{\partial^{3} P_{l}}{\partial x^{3}}=0
\end{aligned}
$$

respectively, with $l=1,2$ and $\bar{l}=3-l$. Since the profile function $P_{l}(x, t)$ can be written in the form $f(x-v t)$ where the function $f$ represents the soliton wave profile depending the type of nonlinearity and $v$ is the soliton speed. From the imaginary part (??) the soliton speed can be obtain as

$$
v=\frac{b_{l} \omega+\alpha_{l}-2 a_{l} \kappa}{1-b_{l} \kappa} .
$$

provided

$$
\gamma_{l}=0
$$

and

$$
2 \eta_{l}+\xi_{l}+3 \lambda_{l}=0 .
$$

It must be noted that the special case where $\alpha_{l}=\xi_{l}=\eta_{l}=\lambda_{l}=\gamma_{l}=0$, one recovers non-dispersive situation that was studied and reported in 2014 [9]. After comparing both resulting values of the soliton speed one can conclude that the coefficients of the linear components of the general system (??)-(??) become

$$
a_{1}=a_{2}, \quad b_{1}=b_{2} \quad \text { and } \quad \alpha_{1}=\alpha_{2} .
$$

Consequently (??) becomes

$$
v=\frac{\alpha+b \omega-2 a \kappa}{1-b \kappa} .
$$

regardless the nonlinearity under consideration, and the real part (??), takes the following new form:

$$
\begin{aligned}
& a \frac{\partial^{2} P_{l}}{\partial x^{2}}+b \frac{\partial^{2} P_{l}}{\partial x \partial t}+[\kappa(\alpha+b \omega-a \kappa)-\omega] P_{l}+\kappa\left(\xi_{l}+\lambda_{l}\right) P_{l}^{3} \\
+\quad & \left\{c_{l} F\left(P_{l}^{2}\right)+d_{l} F\left(P_{\bar{l}}^{2}\right)\right\} P_{l}=Q_{l} P_{\bar{l}}
\end{aligned}
$$

This real part will be considered in the following subsections with two different forms of the functional $F$, they are Kerr (cubic nonlinearity) and power laws.

\subsection{KERR LAW NONLINEARITY}

For Kerr law nonlinearity $F(s)=s$, thus the equations describing the dispersive soliton propagation in magneto-optic waveguides can be rewritten as

$$
\begin{gathered}
\quad i q_{t}+i \alpha q_{x}+a q_{x x}+b q_{x t}+\left(c_{1}|q|^{2}+d_{1}|r|^{2}\right) q \\
+\quad i \lambda_{1}\left(|q|^{2} q\right)_{x}+i \xi_{1}|q|^{2} q_{x}+i \eta_{1}\left(|q|^{2}\right)_{x} q=Q_{1} r \\
\quad i r_{t}+i \alpha r_{x}+a r_{x x}+b r_{x t}+\left(c_{2}|r|^{2}+d_{2}|q|^{2}\right) r \\
+i \lambda_{2}\left(|r|^{2} r\right)_{x}+i \xi_{2}|r|^{2} r_{x}+i \eta_{2}\left(|r|^{2}\right)_{x} r=Q_{2} q
\end{gathered}
$$


in view of (??)-(??). As a result of this nonlinearity, the real part (??), becomes

$$
\begin{gathered}
\quad a \frac{\partial^{2} P_{l}}{\partial x^{2}}+b \frac{\partial^{2} P_{l}}{\partial x \partial t}+[\kappa(\alpha+b \omega-a \kappa)-\omega] P_{l} \\
+\quad\left[c_{l}+\kappa\left(\xi_{l}+\lambda_{l}\right)\right] P_{l}^{3}+d_{l} P_{l} P_{\bar{l}}^{2}=Q_{l} P_{\bar{l}}
\end{gathered}
$$

with $l=1,2$ and $\bar{l}=3-l$.

\subsubsection{BRIGHT SOLITONS}

To investigate the dynamics of bright soliton propagation, one integrate (??) considering the starting hypothesis for $P_{l}$ :

$$
P_{l}=A_{l} \operatorname{sech}^{p_{l}} \tau
$$

where

$$
\tau=B(x-v t)
$$

In (??) $A_{l}$ stands for the pulse amplitude while $B$ is the corresponding inverse width. The substitution of (??) into (??) leads to

$$
\begin{aligned}
& \left\{[\kappa(\alpha+b \omega-a \kappa)-\omega]+p_{l}^{2}(a-b v) B^{2}\right\} A_{l} \operatorname{sech}^{p_{l}} \tau \\
& -p_{l}\left(1+p_{l}\right)(a-b v) A_{l} B^{2} \operatorname{sech}^{p_{l}+2} \tau \\
& +\left[c_{l}+\kappa\left(\xi_{l}+\lambda_{l}\right)\right] A_{l}^{3} \operatorname{sech}^{3 p_{l}} \tau \\
& +d_{l} A_{l} A_{\bar{l}}^{2} \operatorname{sech}^{p_{l}+2 p_{\bar{l}}} \tau-Q_{l} A_{\bar{l}} \operatorname{sech}^{p_{\bar{l}}} \tau=0
\end{aligned}
$$

after simplification. After applying the balancing principle in (??) one obtains

$$
3 p_{l}=2+p_{l}
$$

leading to

$$
p_{l}=1
$$

for $l=1,2$. Then, setting the coefficients of the resulting linearly independent functions $\operatorname{sech}^{j} \tau$ to zero, for $j=1,3$ leads to the speed and wave numbers of the bright solitons as

$$
v=\frac{2 a B^{2}-\left[c_{l}+\kappa\left(\lambda_{l}+\xi_{l}\right)\right] A_{l}^{2}-d_{l} A_{l}^{2}}{2 b B^{2}}
$$

and

$$
\omega=\frac{2 Q_{l} A_{\bar{l}}-\left[c_{l}+\kappa\left(\lambda_{l}+\xi_{l}\right)\right] A_{l}^{3}-\left[2 \kappa(\alpha-a \kappa)+d_{l} A_{\bar{l}}^{2}\right] A_{l}}{2(b \kappa-1) A_{l}}
$$

respectively, whenever $b \kappa \neq 1$ and $b B \neq 0$ are satisfied. In addition, one can notice by equating both alternative expressions for the soliton speed $v$ for $l=1,2$ in (??) the relation between the amplitudes

$$
\frac{A_{1}}{A_{2}}=\sqrt{\frac{c_{2}+\kappa\left(\lambda_{2}+\xi_{2}\right)-d_{1}}{c_{1}+\kappa\left(\lambda_{1}+\xi_{1}\right)-d_{2}}}
$$


constrained by

$$
\left\{c_{2}+\kappa\left(\lambda_{2}+\xi_{2}\right)-d_{1}\right\}\left\{c_{1}+\kappa\left(\lambda_{1}+\xi_{1}\right)-d_{2}\right\}>0 .
$$

Following a similar procedure, equating the two expressions for the soliton wave numbers from (??) for $l=1,2$ one obtain a connection between the amplitudes of bright solitons in the two components as

$$
2 Q_{1} A_{2}^{2}-\left[c_{1}+\kappa\left(\lambda_{1}+\xi_{1}\right)-d_{2}\right] A_{1}^{3} A_{2}=2 Q_{2} A_{1}^{2}-\left[c_{2}+\kappa\left(\lambda_{2}+\xi_{2}\right)-d_{1}\right] A_{2}^{3} A_{1}
$$

With the help of (??), by comparing (??) and (??) it is possible to obtain

$$
A_{l}=B \sqrt{-\frac{2[a(1+b \kappa)-b(\alpha+b \omega)]\left[c_{\bar{l}}+\kappa\left(\lambda_{\bar{l}}+\xi_{\bar{l}}\right)-d_{l}\right]}{(b \kappa-1)\left[\left(c_{l}+\kappa\left(\lambda_{l}+\xi_{l}\right)\right)\left(c_{\bar{l}}+\kappa\left(\lambda_{\bar{l}}+\xi_{\bar{l}}\right)\right)-d_{l} d_{\bar{l}}\right]}}
$$

for $l=1,2$ and $\bar{l}=3-l$ whenever the inequality

$$
\begin{gathered}
\quad[a(1+b \kappa)-b(\alpha+b \omega)]\left[c_{\bar{l}}+\kappa\left(\lambda_{\bar{l}}+\xi_{\bar{l}}\right)-d_{l}\right] \\
\times(b \kappa-1)\left[\left(c_{l}+\kappa\left(\lambda_{l}+\xi_{l}\right)\right)\left(c_{\bar{l}}+\kappa\left(\lambda_{\bar{l}}+\xi_{\bar{l}}\right)\right)-d_{l} d_{\bar{l}}\right]<0 .
\end{gathered}
$$

holds. Also, by substituting (??) into (??) for both $l=1$ and $l=2$ one get two possible expressions for the frequency:

$$
\kappa=\frac{b(\alpha+b \omega)-a}{a b}
$$

and

$$
\kappa=\frac{\left(d_{2}-c_{1}\right) Q_{1}-\left(d_{1}-c_{2}\right) Q_{2}}{\left(\lambda_{1}+\xi_{1}\right) Q_{1}-\left(\lambda_{2}+\xi_{2}\right) Q_{2}}
$$

conditioned to

$$
\begin{aligned}
a b & \neq 0 \\
\left(\lambda_{1}+\xi_{1}\right) Q_{1} & \neq\left(\lambda_{2}+\xi_{2}\right) Q_{2}
\end{aligned}
$$

respectively. Thus, we can conclude that the bright soliton solution for the system describing dispersive magneto-optic waveguides is given by

$$
\begin{aligned}
& q(x, t)=A_{1} \operatorname{sech}[B(x-v t)] e^{i(-\kappa x+\omega t+\theta)} \\
& r(x, t)=A_{2} \operatorname{sech}[B(x-v t)] e^{i(-\kappa x+\omega t+\theta)}
\end{aligned}
$$

where parameters and corresponding constraints are discussed above.

\subsubsection{DARK SOLITONS}

In order to explore the propagation of dark solitons, one integrate (??) adopting the ansatz

$$
P_{l}=A_{l} \tanh ^{p_{l}} \tau
$$

where the argument $\tau$ is as given in (??), $A_{l}$ still describes the pulse amplitude while $p_{l}$ is a free parameter to be calculated throughout the next few lines. The substitution of (??) into (??) leads 
to

$$
\begin{aligned}
& \left\{[\kappa(\alpha+b \omega-a \kappa)-\omega]-2 p_{l}^{2}(a-b v) B^{2}\right\} A_{l} \tanh ^{p_{l}} \tau \\
& +p_{l}\left(p_{l}-1\right)(a-b v) A_{l} B^{2} \tanh ^{p_{l}-2} \tau \\
& +p_{l}\left(p_{l}+1\right)(a-b v) A_{l} B^{2} \tanh ^{p_{l}+2} \tau \\
& +\left[c_{l}+\kappa\left(\xi_{l}+\lambda_{l}\right)\right] A_{l}^{3} \tanh ^{3 p_{l}} \tau \\
& +d A_{l} A_{\bar{l}}^{2} \tanh ^{p_{l}+2 p_{\bar{l}}} \tau-Q_{l} A_{\bar{l}} \tanh ^{p_{\bar{l}}} \tau=0
\end{aligned}
$$

The delicate balance between dispersion and nonlinearity, as well as the standalone linearly independent function $\tanh ^{p_{l}-2} \tau$ drives one to retrieve the value of $p_{l}$ as in (??). Thus, setting to zero the coefficients of the linearly independent functions $\tanh ^{j} \tau$ for $j=1,3$ leads to

$$
v=\frac{2 a B^{2}+\left[c_{l}+\kappa\left(\lambda_{l}+\xi_{l}\right)\right] A_{l}^{2}+d_{l} A_{\bar{l}}^{2}}{2 b B^{2}}
$$

and

$$
\omega=\frac{Q_{l} A_{\bar{l}}-\left[c_{l}+\kappa\left(\lambda_{l}+\xi_{l}\right)\right] A_{l}^{3}-\left[\kappa(\alpha-a \kappa)+d_{l} A_{\bar{l}}^{2}\right] A_{l}}{(b \kappa-1) A_{l}} .
$$

Notice in (??), after comparing the two resulting waves speeds $v$ for $l=1,2$ the quotient between amplitudes implies the same relation as given in (??) and (??). Similarly, comparing the two resulting wave functions $\omega$ in (??) the following identity arise:

$$
Q_{1} A_{2}^{2}+\left[c_{1}+\kappa\left(\lambda_{1}+\xi_{1}\right)-d_{2}\right] A_{1}^{3} A_{2}=Q_{2} A_{1}^{2}+\left[c_{2}+\kappa\left(\lambda_{2}+\xi_{2}\right)-d_{1}\right] A_{2}^{3} A_{1}
$$

By considering (??) and comparing the two possible expressions for the speed (??) and (??) to each other for either value of $l$, yields

$$
A_{l}=B \sqrt{\frac{2[a(1+b \kappa)-b(\alpha+b \omega)]\left[c_{\bar{l}}+\kappa\left(\lambda_{\bar{l}}+\xi_{\bar{l}}\right)-d_{l}\right]}{(b \kappa-1)\left[\left(c_{l}+\kappa\left(\lambda_{l}+\xi_{l}\right)\right)\left(c_{\bar{l}}+\kappa\left(\lambda_{\bar{l}}+\xi_{\bar{l}}\right)\right)-d_{l} d_{\bar{l}}\right]}}
$$

as long as

$$
\begin{gathered}
{[a(1+b \kappa)-b(\alpha+b \omega)]\left[c_{\bar{l}}+\kappa\left(\lambda_{\bar{l}}+\xi_{\bar{l}}\right)-d_{l}\right]} \\
\times(b \kappa-1)\left[\left(c_{l}+\kappa\left(\lambda_{l}+\xi_{l}\right)\right)\left(c_{\bar{l}}+\kappa\left(\lambda_{\bar{l}}+\xi_{\bar{l}}\right)\right)-d_{l} d_{\bar{l}}\right]>0 .
\end{gathered}
$$

stay valid. By substituting (??) into (??) for both $l=1$ and $l=2$ one recalculate (??)(??).Therefore, the dark soliton solution for the system describing dispersive magneto-optic waveguides with cubic nonlinearity (??)-(??) is given by

$$
\begin{aligned}
& q(x, t)=A_{1} \tanh [B(x-v t)] e^{i(-\kappa x+\omega t+\theta)} \\
& r(x, t)=A_{2} \tanh [B(x-v t)] e^{i(-\kappa x+\omega t+\theta)}
\end{aligned}
$$

where the soliton amplitude is described by (??), the speed and the wave numbers are (??) and (??), while the frequency is either described by (??) or (??), with corresponding constraints.

\subsubsection{SINGULAR SOLITONS (TYPE-I)}

This part is devoted to explore the type-I of singular soliton solutions of the form

$$
P_{l}=A_{l} \operatorname{csch}^{p_{l}} \tau
$$


where $A_{l}$ describes the pulse amplitude, the parameter $p_{l}$ will be calculated by balancing nonlinearity and dispersion, and the argument $\tau$ has been defined in (??). Direct substitution of (??) into (??) results in

$$
\left.\begin{array}{l}
\left\{[\kappa(\alpha+b \omega-a \kappa)-\omega]+p_{l}^{2}(a-b v) B^{2}\right\} A_{l} \operatorname{csch}^{p_{l}} \tau \\
+p_{l}\left(1+p_{l}\right)(a-b v) A_{l} B^{2} \operatorname{csch}^{p_{l}+2} \tau \\
+\left[c_{l}+\kappa\left(\xi_{l}+\lambda_{l}\right)\right] A_{l}^{3} \operatorname{csch}^{3 p_{l}} \tau \\
+d A_{l} A_{\bar{l}}^{2} \operatorname{csch}^{p_{l}+2 p_{\bar{l}}} \tau-Q_{l} A_{\bar{l}} \operatorname{csch}^{p_{\bar{l}}} \tau=0 .
\end{array}\right\}
$$

After the balancing procedure one retrieve the value of the parameter $p_{l}$ as in (??). The same can be obtain from the coefficient of the stand alone element $\operatorname{csch}^{p_{l}-2} \tau$. After substituting $p=1$, for $l=1,2$ in (??), setting the coefficients of the linearly independent functions $\operatorname{csch}^{j} \tau$ for $j=1,3$ to zero allow to retrieve the soliton speed as for dark soliton (??), while the wave numbers become

$$
\omega=\frac{2 Q_{l} A_{\bar{l}}+\left[c_{l}+\kappa\left(\lambda_{l}+\xi_{l}\right)\right] A_{l}^{3}-\left[2 \kappa(\alpha-a \kappa)-d_{l} A_{\bar{l}}^{2}\right] A_{l}}{2(b \kappa-1) A_{l}} .
$$

Equating the resulting two expressions for the soliton wave in the last equation (??) for $l=1,2$ gives

$$
2 Q_{1} A_{2}^{2}+\left[c_{1}+\kappa\left(\lambda_{1}+\xi_{1}\right)-d_{2}\right] A_{1}^{3} A_{2}=2 Q_{2} A_{1}^{2}+\left[c_{2}+\kappa\left(\lambda_{2}+\xi_{2}\right)-d_{1}\right] A_{2}^{3} A_{1} .
$$

Thus, considering (??) and equating the two possible expressions for the speed (??) and (??) to each other for either $l=1$ or $l=2$ yield (??) constrained by (??). Also substituting (??) and (??) into (??) provides the same expressions for $\kappa$ and corresponding solvability conditions (??)-(??). Therefore, the type-I singular soliton solution for the system (??)-(??) is

$$
\begin{aligned}
& q(x, t)=A_{1} \operatorname{csch}[B(x-v t)] e^{i(-\kappa x+\omega t+\theta)} \\
& r(x, t)=A_{2} \operatorname{csch}[B(x-v t)] e^{i(-\kappa x+\omega t+\theta)} .
\end{aligned}
$$

This type-I singular soliton solution will persist whenever the corresponding constrains above discussed are satisfied.

\subsubsection{SINGULAR SOLITONS (TYPE-II)}

To study the second type of singular solitons for the system (??)-(??), we adopt an ansatz of the form

$$
P_{l}=A_{l} \operatorname{coth}^{p_{l}} \tau
$$

where the meaning of the amplitude $A_{l}$, the argument $\tau$ and the parameter $p_{l}$ is the same as for the previous starting hypothesis in Kerr law. The substitution of the hypothesis (??) into (??) leads to

$$
\left.\begin{array}{l}
\left\{[\kappa(\alpha+b \omega-a \kappa)-\omega]-2 p_{l}^{2}(a-b v) B^{2}\right\} A_{l} \operatorname{coth}^{p_{l}} \tau \\
+p_{l}\left(p_{l}-1\right)(a-b v) A_{l} B^{2} \operatorname{coth}^{p_{l}-2} \tau \\
+p_{l}\left(p_{l}+1\right)(a-b v) A_{l} B^{2} \operatorname{coth}^{p_{l}+2} \tau \\
+\left[c_{l}+\kappa\left(\xi_{l}+\lambda_{l}\right)\right] A_{l}^{3} \operatorname{coth}^{3 p_{l}} \tau \\
+d A_{l} A_{\bar{l}}^{2} \operatorname{coth}^{p_{l}+2 p_{\bar{l}}} \tau-Q_{l} A_{\bar{l}} \operatorname{coth}^{p_{\bar{l}}} \tau=0 .
\end{array}\right\}
$$


Balancing principle, as well as the coefficient of the stand alone element $\operatorname{coth}^{p_{l}-2} \tau$ yields the same value of the parameter $p_{l}$ as in (??). The substitution of the resulting value of $p_{l}$ into (??) leads to the same results as for dark solitons (??)-(??) along with corresponding conditions. Therefore, type-I singular soliton solution for the system (??)-(??) is of the form

$$
\begin{aligned}
& q(x, t)=A_{1} \operatorname{coth}[B(x-v t)] e^{i(-\kappa x+\omega t+\theta)} \\
& r(x, t)=A_{2} \operatorname{coth}[B(x-v t)] e^{i(-\kappa x+\omega t+\theta)} .
\end{aligned}
$$

where the corresponding parameters as well as constraints are the same as for dark solitons.

\subsection{POWER LAW NONLINEARITY}

This section is devoted to study the system (??)-(??) with power law nonlinearity. Under this nonlinearity the nonlinear functional is of the general form $F(s)=s^{n}$ where $n$ represents the power law nonlinearity parameter. For wave stability purposes we are restricted to $0<n<2$, where $n \neq 2$ in order to avoid self-focusing singularity. Stating that, the original system (??)-(??) is rewritten as

$$
\begin{gathered}
i q_{t}+i \alpha_{1} q_{x}+a_{1} q_{x x}+b_{1} q_{x t}+\left(c_{1}|q|^{2 n}+d_{1}|r|^{2 n}\right) q \\
+\quad i \gamma_{1} q_{x x x}+i \lambda_{1}\left(|q|^{2 n} q\right)_{x}+i \xi_{1}|q|^{2 n} q_{x}+i \eta_{1}\left(|q|^{2 n}\right)_{x} q=Q_{1} r \\
\quad i r_{t}+i \alpha_{2} r_{x}+a_{2} r_{x x}+b_{2} r_{x t}+\left(c_{2}|r|^{2 n}+d_{2}|q|^{2 n}\right) r \\
+\quad i \gamma_{2} r_{x x x}+i \lambda_{2}\left(|r|^{2 n} r\right)_{x}+i \xi_{2}|r|^{2 n} r_{x}+i \eta_{2}\left(|r|^{2 n}\right)_{x} r=Q_{2} q
\end{gathered}
$$

The substitution of (??) and (??) into (??) and (??) transform the real part (??) into

$$
\begin{aligned}
& a_{l} \frac{\partial^{2} P_{l}}{\partial x^{2}}+b_{l} \frac{\partial^{2} P_{l}}{\partial x \partial t}+\left[\kappa\left(\alpha_{l}+b \omega-a_{l} \kappa\right)-\omega-\gamma_{l} \kappa^{3}\right] P_{l} \\
+\quad & \kappa\left(\xi_{l}+\lambda_{l}\right) P_{l}^{2 n+1}+\left(c_{l} P_{l}^{2 n}+d_{l} P_{\bar{l}}^{2 n}\right) P_{l}=Q_{l} P_{\bar{l}}
\end{aligned}
$$

and the imaginary part takes the form

$$
\begin{aligned}
& \left(1-b_{l} \kappa\right) \frac{\partial P_{l}}{\partial t}+\left(b_{l} \omega+\alpha_{l}-2 a_{l} \kappa-3 \gamma_{l} \kappa^{2}\right) \frac{\partial P_{l}}{\partial x} \\
+ & {\left.\left[2 n \eta_{l}+\xi_{l}+(2 n+1) \lambda_{l}\right)\right] P_{l}^{2} \frac{\partial P_{l}}{\partial x}+\gamma_{l} \frac{\partial^{3} P_{l}}{\partial x^{3}}=0 }
\end{aligned}
$$

From the last equation (??) one can retrieve (??) as long as (??) and

$$
2 n \eta_{l}+\xi_{l}+(2 n+1) \lambda_{l}=0 .
$$

In this case it is also possible to get (??), and consequently (??). In view of this constraints the real part (??) reduces to

$$
\begin{aligned}
& a \frac{\partial^{2} P_{l}}{\partial x^{2}}+b \frac{\partial^{2} P_{l}}{\partial x \partial t}+\left[\kappa\left(\alpha+b \omega-a_{l} \kappa\right)-\omega\right] P_{l} \\
+ & {\left[c_{l}+\kappa\left(\xi_{l}+\lambda_{l}\right)\right] P_{l}^{2 n+1}+d_{l} P_{\bar{l}}^{2 n} P_{l}=Q_{l} P_{\bar{l}} }
\end{aligned}
$$


The ansatz approach will be applied in the next few subsections to the real part equation (??) to analyze four different forms of soliton solutions.

\subsubsection{BRIGHT SOLITONS}

To study bright soliton solution on the system (??)-(??) we adopt the same starting hypothesis as for cubic nonlinearity given by (??) jointly with (??). After substituting (??) into (??) we get

$$
\left.\begin{array}{l}
\left\{[\kappa(\alpha+b \omega-a \kappa)-\omega]+p_{l}^{2}(a-b v) B^{2}\right\} A_{l} \operatorname{sech}^{p_{l}} \tau \\
-p_{l}\left(1+p_{l}\right)(a-b v) A_{l} B^{2} \operatorname{sech}^{p_{l}+2} \tau \\
+\left[c_{l}+\kappa\left(\xi_{l}+\lambda_{l}\right)\right] A_{l}^{2 n+1} \operatorname{sech}^{(2 n+1) p_{l}} \tau \\
+d_{l} A_{l} A_{\bar{l}}^{2 n} \operatorname{sech}^{p_{l}+2 n p_{\bar{l}}} \tau-Q_{l} A_{\bar{l}} \operatorname{sech}^{p_{\bar{l}}} \tau=0
\end{array}\right\}
$$

The balance between nonlinearity and dispersion leads to

$$
(2 n+1) p_{l}=p_{l}+2
$$

from which

$$
p_{l}=\frac{1}{n}
$$

for $l=1$ and $l=2$. Then, after the substitution of (??) into (??), and setting the coefficients of the linearly independent functions $\operatorname{sech}^{j} \tau$ to zero for $j=\frac{1}{n}, \frac{2 n+1}{n}$ one get

$$
v=\frac{(n+1) a B^{2}-n^{2}\left[c_{l}+\kappa\left(\lambda_{l}+\xi_{l}\right)\right] A_{l}^{2 n}-n^{2} d_{l} A_{\bar{l}}^{2 n}}{(n+1) b B^{2}}
$$

and

$$
\omega=\frac{(n+1) Q_{l} A_{\bar{l}}-\left[c_{l}+\kappa\left(\lambda_{l}+\xi_{l}\right)\right] A_{l}^{2 n+1}-\left[(n+1) \kappa(\alpha-a \kappa)+d_{l} A_{\bar{l}}^{2 n}\right] A_{l}}{2(b \kappa-1) A_{l}}
$$

respectively, as long as $b \kappa \neq 1$ and $b B \neq 0$ are satisfied. Notice by equating both alternative expressions for the soliton speed $v$ for $l=1,2$ in (??) the relation between the amplitudes takes the form

$$
\frac{A_{1}}{A_{2}}=\left(\frac{c_{2}+\kappa\left(\lambda_{2}+\xi_{2}\right)-d_{1}}{c_{1}+\kappa\left(\lambda_{1}+\xi_{1}\right)-d_{2}}\right)^{\frac{1}{2 n}}
$$

where $l=1,2$ and $\bar{l}=3-l$, subject to condition (??). In a similar manner, equating the two expressions for the soliton wave numbers from (??) for $l=1,2$ the connection between the amplitudes of bright solitons in the two components is obtained as

$$
\begin{aligned}
& (n+1) Q_{1} A_{2}^{2}-\left[c_{1}+\kappa\left(\lambda_{1}+\xi_{1}\right)-d_{2}\right] A_{1}^{2 n+1} A_{2} \\
& \quad=(n+1) Q_{2} A_{1}^{2}-\left[c_{2}+\kappa\left(\lambda_{2}+\xi_{2}\right)-d_{1}\right] A_{2}^{2 n+1} A_{1} .
\end{aligned}
$$

With the help of (??), equating (??) and (??) either for $l=1$ or $l=2$ yield

$$
A_{l}=\left[-\frac{(n+1) B^{2}[a(1+b \kappa)-b(\alpha+b \omega)]\left[c_{\bar{l}}+\kappa\left(\lambda_{\bar{l}}+\xi_{\bar{l}}\right)-d_{l}\right]}{n^{2}(b \kappa-1)\left[\left(c_{l}+\kappa\left(\lambda_{l}+\xi_{l}\right)\right)\left(c_{\bar{l}}+\kappa\left(\lambda_{\bar{l}}+\xi_{\bar{l}}\right)\right)-d_{l} d_{\bar{l}}\right]}\right]^{\frac{1}{2 n}}
$$


whenever the inequality (??) holds.Therefore, the bright soliton solution for the system describing dispersive magneto-optic waveguides is given by

$$
\begin{aligned}
& q(x, t)=A_{1} \operatorname{sech}^{\frac{1}{n}}[B(x-v t)] e^{i(-\kappa x+\omega t+\theta)} \\
& r(x, t)=A_{2} \operatorname{sech}^{\frac{1}{n}}[B(x-v t)] e^{i(-\kappa x+\omega t+\theta)}
\end{aligned}
$$

The persistence of these bright soliton solution will be dictated by the conditions discussed above.

\subsubsection{DARK SOLITONS}

For dark solitons the ansatz to be taken is the same as in (??). Consequently the real part equation (??) reduces to

$$
\begin{aligned}
& \left\{[\kappa(\alpha+b \omega-a \kappa)-\omega]-2 p_{l}^{2}(a-b v) B^{2}\right\} A_{l} \tanh ^{p_{l}} \tau \\
& +p_{l}\left(p_{l}-1\right)(a-b v) A_{l} B^{2} \tanh ^{p_{l}-2} \tau \\
& +p_{l}\left(p_{l}+1\right)(a-b v) A_{l} B^{2} \tanh ^{p_{l}+2} \tau \\
& +\left[c_{l}+\kappa\left(\xi_{l}+\lambda_{l}\right)\right] A_{l}^{2 n+1} \tanh ^{(2 n+1) p_{l}} \tau \\
& +d A_{l} A_{\bar{l}}^{2 n} \tanh ^{p_{l}+2 n p_{\bar{l}}} \tau-Q_{l} A_{\bar{l}} \tanh ^{p_{\bar{l}}} \tau=0
\end{aligned}
$$

In this case the balancing principle allow to retrieve the value of the parameter $p_{l}$ as in (??). However, the standalone element $\tanh ^{p_{l}-2} \tau$ leads to $p_{l}$ as the one given in (??) forcing $n=1$. Consequently the system (??)-(??) reduces to (??)-(??), and the dark soliton solution will exist in dispersive magneto-optic waveguides whenever the power law nonlinearity collapses to Kerr law. Therefore, the results for this subsection will be the same as of dark solitons for cubic nonlinearity (??)-(??) as well.

\subsubsection{SINGULAR SOLITONS (TYPE-I)}

To study the first type of singular soliton solution of the system (??)-(??), the guess function (??) is readopted. The same reduces the real portion equation (??) into

$$
\left.\begin{array}{l}
\left\{[\kappa(\alpha+b \omega-a \kappa)-\omega]+p_{l}^{2}(a-b v) B^{2}\right\} A_{l} \operatorname{csch}^{p_{l}} \tau \\
+p_{l}\left(1+p_{l}\right)(a-b v) A_{l} B^{2} \operatorname{csch}^{p_{l}+2} \tau \\
+\left[c_{l}+\kappa\left(\xi_{l}+\lambda_{l}\right)\right] A_{l}^{2 n+1} \operatorname{csch}^{(2 n+1) p_{l}} \tau \\
+d A_{l} A_{\bar{l}}^{2} \operatorname{csch}^{p_{l}+2 n p_{\bar{l}}} \tau-Q_{l} A_{\bar{l}} \operatorname{csch}^{p_{\bar{l}}} \tau=0 .
\end{array}\right\}
$$

Appropriate balance between dispersion and nonlinear terms yield the value of $p_{l}$ obtained before in (??). Thus, from (??), from the coefficients of the linearly independent functions $\operatorname{csch}^{j} \tau$ for $j=\frac{1}{n}$ and $j=\frac{2 n+1}{n}$ the soliton's speed and wave numbers take the form

$$
v=\frac{(n+1) a B^{2}+n^{2}\left[c_{l}+\kappa\left(\lambda_{l}+\xi_{l}\right)\right] A_{l}^{2 n}+n^{2} d_{l} A_{l}^{2 n}}{(n+1) b B^{2}}
$$

and

$$
\omega=\frac{(n+1) Q_{l} A_{\bar{l}}+\left[c_{l}+\kappa\left(\lambda_{l}+\xi_{l}\right)\right] A_{l}^{2 n+1}-\left[(n+1) \kappa(\alpha-a \kappa)-d_{l} A_{\bar{l}}^{2 n}\right] A_{l}}{2(b \kappa-1) A_{l}}
$$


Next, equating the expressions of $v$ for $l=1,2$ in (??) leads to the quotient (??), while performing a similar procedure for $\omega$ in (??) yields the identity

$$
\begin{aligned}
& (n+1) Q_{1} A_{2}^{2}+\left[c_{1}+\kappa\left(\lambda_{1}+\xi_{1}\right)-d_{2}\right] A_{1}^{2 n+1} A_{2} \\
& \quad=(n+1) Q_{2} A_{1}^{2}+\left[c_{2}+\kappa\left(\lambda_{2}+\xi_{2}\right)-d_{1}\right] A_{1} A_{2}^{2 n+1} .
\end{aligned}
$$

For this kind of soliton and under power law nonlinearity, equating (??) and (??) either for $l=1$ or $l=2$ yield

$$
A_{l}=\left[\frac{(n+1) B^{2}[a(1+b \kappa)-b(\alpha+b \omega)]\left[c_{\bar{l}}+\kappa\left(\lambda_{\bar{l}}+\xi_{\bar{l}}\right)-d_{l}\right]}{n^{2}(b \kappa-1)\left[\left(c_{l}+\kappa\left(\lambda_{l}+\xi_{l}\right)\right)\left(c_{\bar{l}}+\kappa\left(\lambda_{\bar{l}}+\xi_{\bar{l}}\right)\right)-d_{l} d_{\bar{l}}\right]}\right]^{\frac{1}{2 n}}
$$

after considering (??), and as long as the inequality (??) holds. Therefore, the type-I singular soliton solution for the system (??)-(??) is

$$
\begin{aligned}
& q(x, t)=A_{1} \operatorname{csch}^{\frac{1}{n}}[B(x-v t)] e^{i(-\kappa x+\omega t+\theta)} \\
& r(x, t)=A_{2} \operatorname{csch}^{\frac{1}{n}}[B(x-v t)] e^{i(-\kappa x+\omega t+\theta)}
\end{aligned}
$$

where the parameters with corresponding constraints are discussed in detail above.

\subsubsection{SINGULAR SOLITONS (TYPE-II)}

The last soliton solution to be considered for the system with power law nonlinearity (??)-(??) is the second type of singular solitons. In order to do so, the starting hypothesis which is given by (??) reduces (??) into

$$
\left.\begin{array}{l}
\left\{[\kappa(\alpha+b \omega-a \kappa)-\omega]-2 p_{l}^{2}(a-b v) B^{2}\right\} A_{l} \operatorname{coth}^{p_{l}} \tau \\
+p_{l}\left(p_{l}-1\right)(a-b v) A_{l} B^{2} \operatorname{coth}^{p_{l}-2} \tau \\
+p_{l}\left(p_{l}+1\right)(a-b v) A_{l} B^{2} \operatorname{coth}^{p_{l}+2} \tau \\
+\left[c_{l}+\kappa\left(\xi_{l}+\lambda_{l}\right)\right] A_{l}^{2 n+1} \operatorname{coth}^{(2 n+1) p_{l}} \tau \\
+d A_{l} A_{\bar{l}}^{2 n} \operatorname{coth}^{p_{l}+2 n p_{\bar{l}}} \tau-Q_{l} A_{\bar{l}} \operatorname{coth}^{p_{\bar{l}}} \tau=0 .
\end{array}\right\}
$$

As expected, the Balancing principle gives the same value for (??). However, the stand alone element $\operatorname{coth}^{p_{l}-2} \tau$ yield $p_{l}=1$. Consequently, the system (??)-(??) reduces to (??)-(??), and the type-II dispersive singular soliton solution will exist in magneto-optic waveguides whenever the power law nonlinearity collapses to Kerr law, as for dark solitons. Therefore, the results for this subsection will be the same as of singular type-II solitons for cubic nonlinearity (??)-(??) as well.

\section{CONCLUSIONS}

This paper obtained exact disperesive optical soliton solution in magneto-optic waveguides for Kerr and power law media. Bright, dark as well as singular optical soliton solutions are recovered. The constraint conditions, for the existence of these solitons, are all listed. The results of this paper are an extension to the ones that was reported in 2014 for non-dispersive optical solitons [9]. It is possible to extend them further. One can consider other laws of nonlinearity, such as anti-cubic nonlinearity, quadratic-cubic law, parabolic law nonlinearity and finally the triple power law nonlinearity. The results of those research will be available in future. 


\section{ACKNOWLEDGEMENT}

The research work of the fourth author (QZ) was funded by National Science Foundation of Hubei Province in China under the grant number 2015CFC891. The authors also declare that there is no conflict of interest. 


\section{References}

[1] A. Bahrami, A. Rostami \& F. Nazari. "MZ-MMI-based all-optical switch using nonlinear coupled waveguides". Optik. Volume 122, Issue 20, 1787-1790. (2011).

[2] A. Biswas \& S. Konar. Introduction to non-Kerr law optical solitons. CRC Press, Boca Raton, FL. (2006).

[3] M. Bouras \& A. Hocini. "Mode conversion in magneto-optic rib waveguide made by silica matrix doped with magnetic nanoparticles". Optics Communications. Volume 363, 138-144. (2016).

[4] C. S Fletcher, J. E Lye, N. P Robins, J. D Close. "A self-locked magneto-optic trap". Optics Communications. Volume 212, Issues 13, Pages 85-88. (2002).

[5] X. Geng \& Y. Lv. "Darboux transformation for an integrable generalization of the nonlinear Schrödinger equation". Nonlinear Dynamics. Volume 69, Number 4, 1621-1630. (2012).

[6] A. H. Kara, A. Biswas \& M. Belic. "Conservation laws for optical solitons in birefringent fibers and magneto-optic waveguides". Optik. Volume 127, Issue 24, 11662-11673. (2016).

[7] S. Kumar, K. Singh \& R. K. Gupta. "Coupled Higgs field equation and Hamiltonian amplitude equation: Lie classical approach and $G^{\prime} / G$-expansion method". Pramana. Volume 79, Number 1, 41-60. (2012).

[8] Y. Miao, B. Liu, K. Zhang, H. Zhang, R. Wang, Y. Liu \& J. Yao. "Magneto-optical tunability of magnetic fluid infiltrated microstructured optical fiber". Optics and Laser Technology. Volume 48, 280-284. (2013).

[9] M. Savescu, A. H. Bhrawy, E. M. Hilal, A. A. Alshaery \& A. Biswas. "Optical solitons in magneto-optic waveguides with spatio-temporal dispersion". Frequenz. Volume 68, Issues 910, 445-451. (2014).

[10] Y. Shen, Y. Lu, X. Yu \& Z. Liu. "Effect of temperature on characteristics of rare earth-doped magneto-optical glass in optical current transducer application". Optik. Volume 126, Issue 23, 3589-3593. (2015). 